\title{
Performance evaluation of new photodetectors for Hyper-Kamiokande
}

\author{
Yusuke Suda $^{* a}$, Yasuhiro Nishimura $^{b}$, Seiko Hirota $^{c}$, Yuji Okajima $^{d}$, Hiroaki Aihara $^{a}$, \\ Masashi Yokoyama ${ }^{a}$, Masato Shiozawa ${ }^{e}$, Yoshinari Hayato ${ }^{e}$, Shoei Nakayama ${ }^{e}$, \\ Hidekazu Tanaka ${ }^{e}$, Tsuyoshi Nakaya $^{c}$, Akimichi Taketa $^{f}$, Yoshihiko Kawai ${ }^{g}$, \\ Takayuki Ohmura ${ }^{g}$, Masatoshi Suzuki ${ }^{g}$ \\ ${ }^{a}$ Department of Physics, The University of Tokyo \\ ${ }^{b}$ Research Center for Cosmic Neutrino, ICRR, The University of Tokyo \\ ${ }^{c}$ Department of Physics, Kyoto University \\ ${ }^{d}$ Department of Physics, Tokyo Institute of Technology \\ ${ }^{e}$ Kamioka Observatory, ICRR, The University of Tokyo \\ ${ }^{f}$ Earthquake Research Institute, The University of Tokyo \\ ${ }^{g}$ Hamamatsu Photonics K.K. \\ E-mail: yusuke@hep.phys.s.u-tokyo.ac.jp
}

\begin{abstract}
We have been developing new photodetectors for Hyper-Kamiokande a proposed next generation megaton-class water Cherenkov detector. Our goal is to develop a high performance photodetector with 20-inch diameter photocathode, which can be used in a water Cherenkov detector for a long time. Two types of new photodetectors are under development; Hybrid Photo-Detectors (HPDs) and photo-multiplier tubes (PMTs) with box-and-line type dynode. High quantum efficiency (QE) photocathode has been developed as a common option for both types. We have been testing 8inch HPDs and high-QE 20-inch PMTs with conventional Venetian blind dynode in a 200-ton water Cherenkov detector since August 2013. We confirmed that the 8-inch HPD shows better performance than PMTs in the tank. Based on the successful development of 8-inch HPDs and high-QE PMTs, we developed prototypes of 20-inch high-QE HPDs and box-and-line PMTs. We measured the basic performance of 20-inch photodetectors and confirmed HPDs and box-and-line PMTs have much better timing and charge resolution than those of Venetian blind PMTs.
\end{abstract}

Technology and Instrumentation in Particle Physics 2014,

2-6 June, 2014

Amsterdam, the Netherlands

* Speaker. 


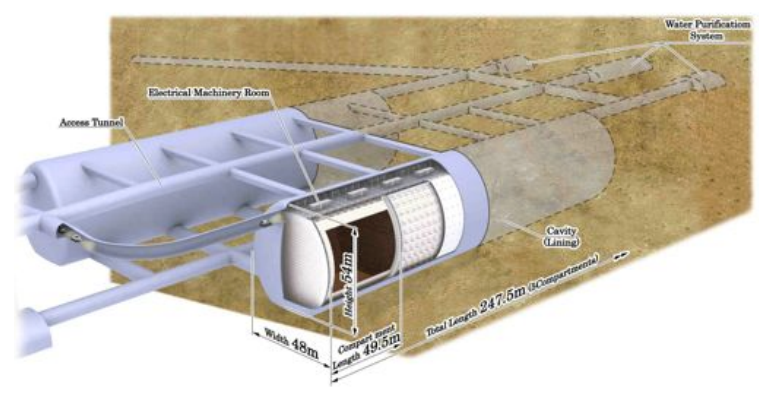

Figure 1: Hyper-Kamiokande.

\section{Hyper-Kamiokande project}

The Hyper-Kamiokande (Hyper-K) is a next generation megaton-class water Cherenkov detector (Fig. 1) [1]. It has rich physics targets such as search for proton decay, study of neutrino properties using neutrino oscillation, detection and study of astrophysical neutrino. The design of Hyper-K is based on the well-proven technologies employed at Super-Kamiokande (Super-K) [2]. The total (fiducial) detector mass is 0.99 (0.56) Mega-ton. In the baseline design, for the inner detector and the outer detector, 99,000 20-inch photo-multiplier tubes (PMTs) and 25,000 8-inch ones are needed, respectively. The photocoverage of the inner detector is $20 \%$ which is a half of that of Super-K.

\section{New photodetectors}

In order to improve the performance of the detector, we have been developing two types of photodetectors. One is box-and-line dynode type PMT and the other is Hybrid Photo-Detector (HPD). A high-quantum efficiency (QE) photocathode is also being developed. In this section, we describe principles of each photodetector and their main features.

\subsection{Box-and-line PMT}

Figure 2 shows the schematic view of Venetian blind dynode PMT used for Super-K (center) and box-and-line PMT (right). For the Venetian blind PMT, electrons can take various drift paths and might miss the dynode. On the other hand, in the box-and-line dynode, electrons take almost unique drift path and tend to hit the first dynode thanks to its large acceptance. Thus, the boxand-line type PMT has better timing resolution, charge resolution and collection efficiency than Venetian blind type PMT.

\subsection{Hybrid Photo-Detector}

An HPD consists of a photocathode and an avalanche diode (AD) as shown in Fig. 3. By applying a high voltage of $8 \mathrm{kV}$, photoelectrons are accelerated to the AD from the photocathode. Then, first, a bombardment gain of around a factor of 1,600 is obtained inside the AD. The avalanche multiplication in the AD gives an additional gain of $30-100$. The total gain of an HPD is about $10^{5}$. We employ a preamplifier to increase the signal from HPD (Fig. 4 and 5). Because 

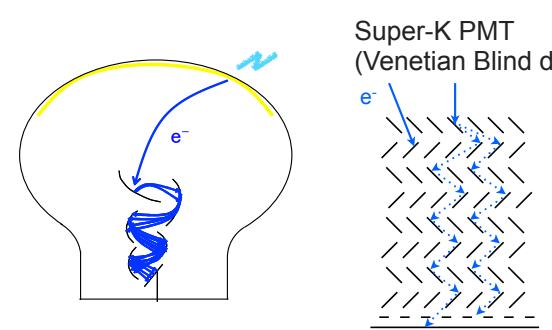

Box\&line PMT

(Box\&line dynode)

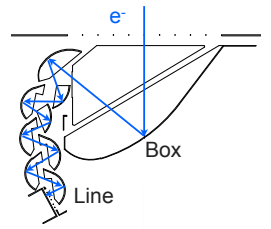

Figure 2: Principle of box-and-line PMT and Super-K PMT. Left is a schematic diagram of PMTs. Center and right figures show the dynode structure of Super-K PMT and box-and-line PMT, respectively.

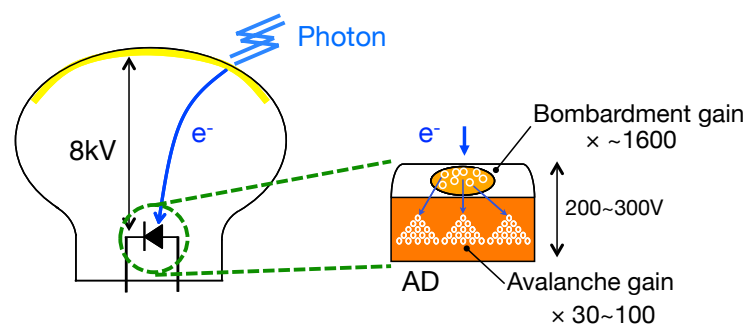

Figure 3: Principle of HPD.
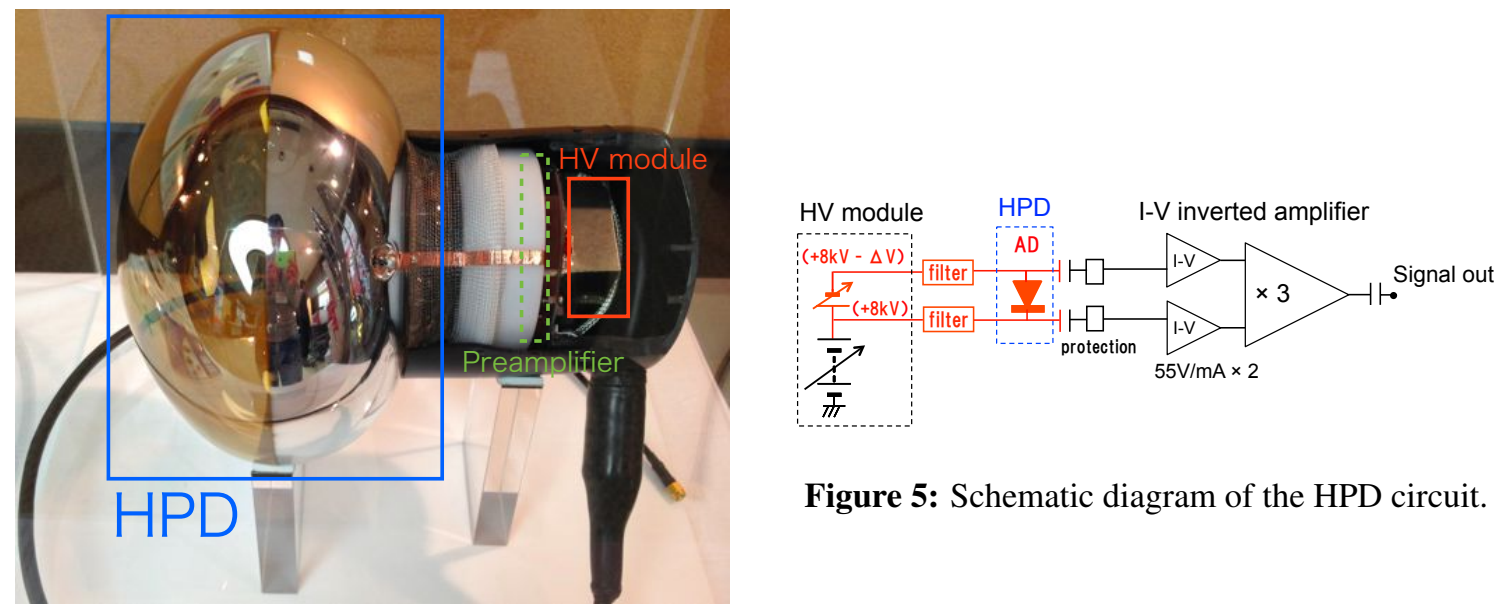

Figure 5: Schematic diagram of the HPD circuit.

Figure 4: 8-inch HPD.

of the short drift path inside the AD and high gain at the first amplification stage, HPD has better timing and charge resolution than that of the conventional PMTs.

\subsection{High-QE photocathode}

We have developed a high-QE photocathode with 20-inch aperture. Around the peak wavelength (400 nm), a QE of about $30 \%$, which is 1.4 times higher than that of Super-K PMT, is achieved (Fig. 6). 


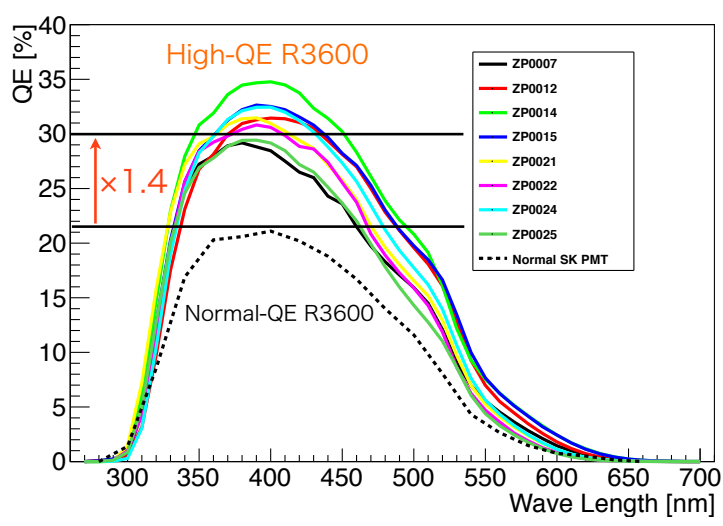

Figure 6: The QE of the Super-K PMT and high-QE version of Super-K PMTs as a function of the wavelength.

\section{R\&D of Hyper-K photodetectors}

Our goal is to develop a 20-inch high performance photodetector that can be used in a water Cherenkov detector for a long time. As a first step of our R\&D, we developed an 8-inch HPD and a high-QE version of 20-inch Super-K PMT and confirmed their performance in air [3]. To evaluate the performance of the 8-inch HPD and the high-QE Super-K PMT, we have been testing eight 8-inch HPDs, five high-QE Super-K PMTs and 227 Super-K PMTs in a 200-ton water Cherenkov detector located in Kamioka mine since August 2013. In parallel to this test, we are developing two types of 20-inch photodetectors with high-QE photocathode, an HPD and a boxand-line PMT based on the technologies of 8-inch HPDs and high-QE Super-K PMTs. After the basic performance evaluation of the prototypes, we will also test these two types of photodetectors in the 200-ton tank.

We report the status of the test in the water tank and the basic performance evaluation of 20-inch prototype photodetectors in the following two sections.

\section{Photodetector test in the 200-ton tank}

The tank used in the test is the 200-ton scale model of the Super-K detector without the outer detector. We use the Super-K old electronics (ATMs) to record the timing and charge from photodetectors [4]. We calibrated the gain of all 8-inch HPDs and high-QE Super-K PMTs as shown in Fig. 7 and 8. Figures 9-11 show the timing and charge resolution of the single photoelectron and the dark rate at $0.5(0.25)$ photoelectron for HPDs (PMTs) of all photodetectors. We confirmed that 8 -inch HPDs have better performance than Super-K PMTs. The gain stability were measured with monitoring single photoelectron peaks for 8-inch HPDs and Xe light pulse for high-QE Super-K PMTs as shown in Fig. 12 and 13. The gain was stable for one month within $2 \%$ and $1 \%$ in root mean square for 8-inch HPDs and high-QE Super-K PMTs, respectively. In the high-QE Super-K PMT stability plot, there is a drop of gain caused by the change of water transparency. 


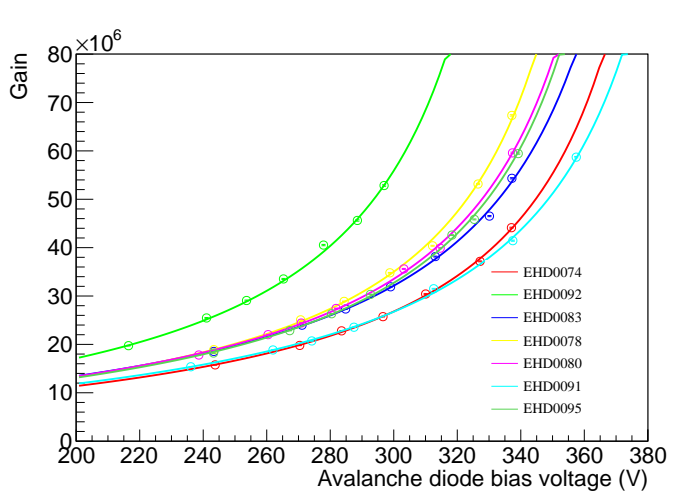

Figure 7: The gain curves of 8-inch HPDs as a function of the $\mathrm{AD}$ bias voltage.

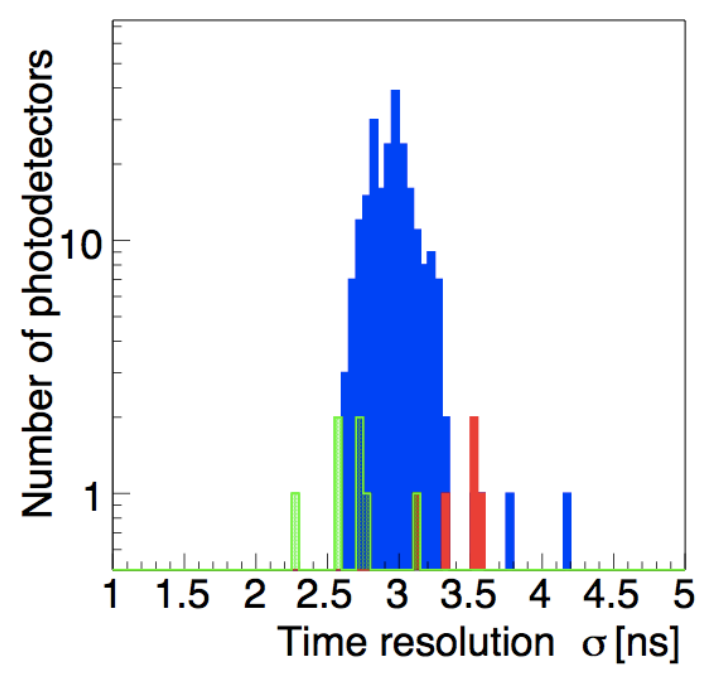

Figure 9: Timing resolution at single photoelectron signal. Green, red and blue for 8-inch HPDs, high-QE Super-K PMTs and Super-K PMTs, respectively.

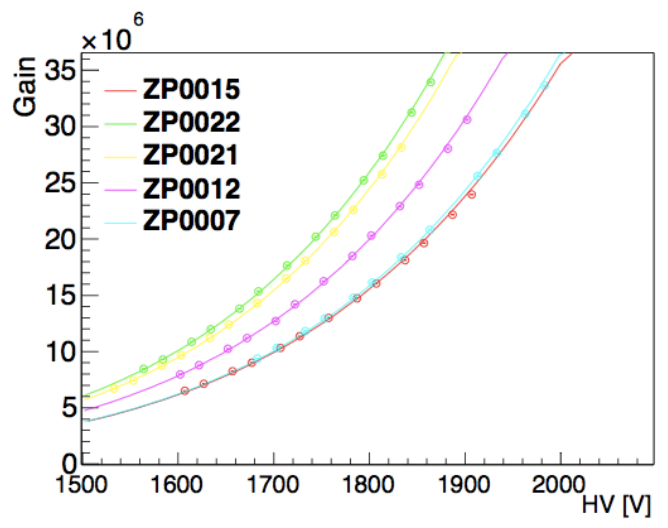

Figure 8: The gain curves of high-QE Super-K PMTs as a function of the high voltage.

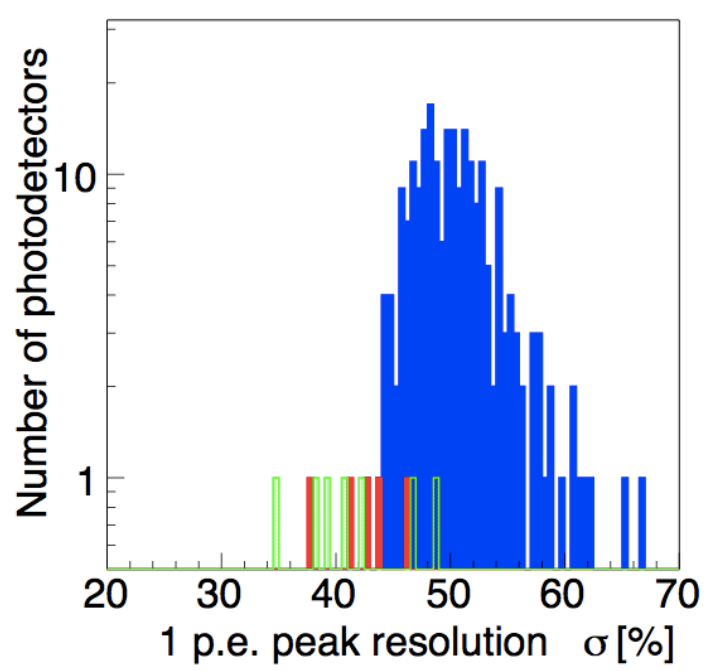

Figure 10: Charge resolution at single photoelectron signal. Green, red and blue for 8-inch HPDs, high-QE Super-K PMTs and Super-K PMTs, respectively.

\section{Basic performance of new 20-inch photodetectors}

We measured the basic performance of the prototype of high-QE 20-inch HPDs and box-andline PMTs. The size of AD used in the 20-inch HPD prototype is $5 \mathrm{~mm}$ in diameter, which is the same as the one used in the 8-inch HPD and smaller than the designed value of $20 \mathrm{~mm}$. Thus, the collection efficiency of the 20-inch HPD prototype is expected to be lower than the design value. Development of a 20-inch HPD with a $20 \mathrm{~mm}$ diameter AD is going on.

Averaged waveforms of the 20-inch high-QE HPD with the preamplifier, the 20-inch high-QE box-and-line PMT and the Super-K PMT are shown in Fig. 14. We can see the response of the box-and-line PMT and the HPD are faster than that of the Super-K PMT. The rise time, fall time between $10 \%$ and $90 \%$ in height and pulse width at $10 \%$ in height are summarized in Table 1 . It 


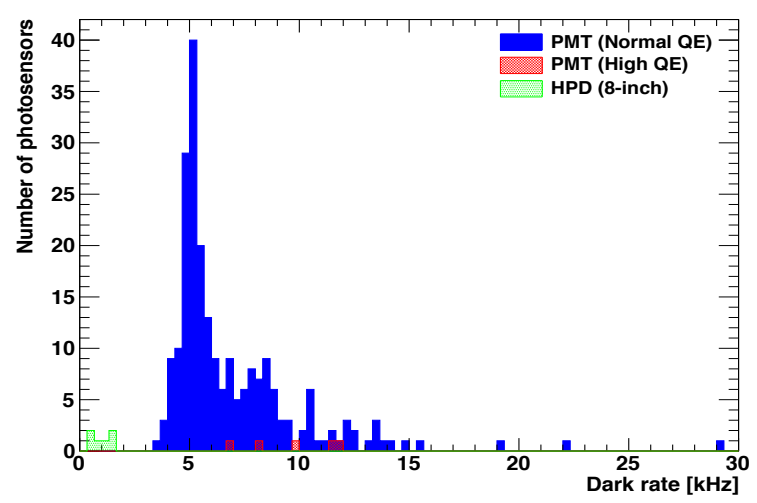

Figure 11: Dark rate at 0.5 (0.25) photoelectron for HPDs (PMTs). Green, red and blue for 8-inch HPDs, high-QE Super-K PMTs and Super-K PMTs, respectively.

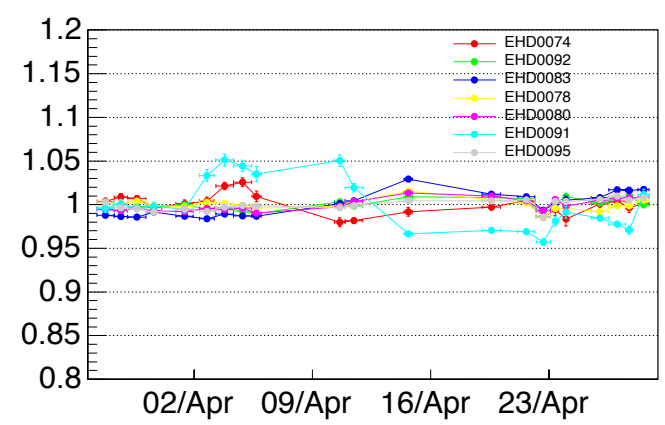

Figure 12: Gain stability of 8-inch HPDs with monitoring single photoelectron peaks.

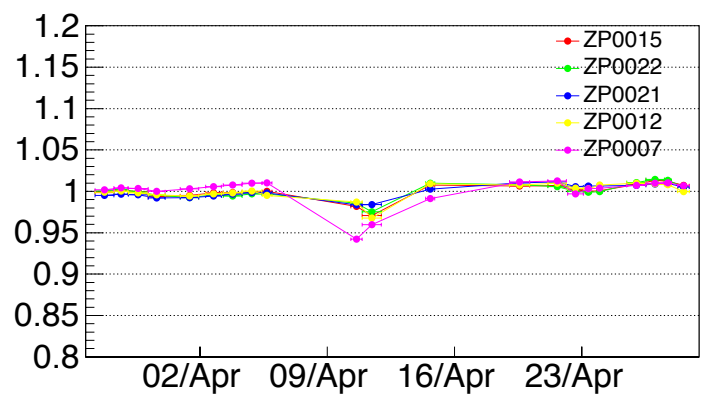

Figure 13: Gain stability of high-QE Super-K PMTs with monitoring Xe light pulse. A drop is due to the change of water transparency.

seems that the HPD response is slower than that of the box-and-line PMT. However, HPD itself has very fast response, the rise time of $1.7 \mathrm{~ns}$ and the fall time of $2.7 \mathrm{~ns}$. The performance of the prototype is limited by the performance of the preamplifier. A new preamplifier is being developed to obtain the best performance.

Figures 15 and 16 show the timing and charge distribution of each photodetector. The timing resolution in sigma is evaluated to be $1.4 \mathrm{~ns}, 1.1 \mathrm{~ns}$ and $2.1 \mathrm{~ns}$ for the HPD, the box-and-line PMT and the Super-K PMT, respectively. The single photoelectron resolution in sigma is $16 \%, 35 \%$ and $53 \%$ for the HPD, the box-and-line PMT and the Super-K PMT, respectively. Both the HPD and the box-and-line PMT show better timing and charge resolution than those of the Super-K PMT. We can clearly see the multi-photoelectron peaks with both the HPD (Fig. 17) and the box-and-line PMT (Fig. 18).

\section{Summary}

We have been developing new photodetectors for Hyper-Kamiokande. Eight-inch HPDs show good performance in the 200-ton water Cherenkov detector. Twenty-inch diameter prototypes of high-QE HPDs and box-and-line PMTs have better timing response, timing resolution and single photoelectron resolution than PMTs used in Super-K. We will install the 20-inch high-QE box-and- 


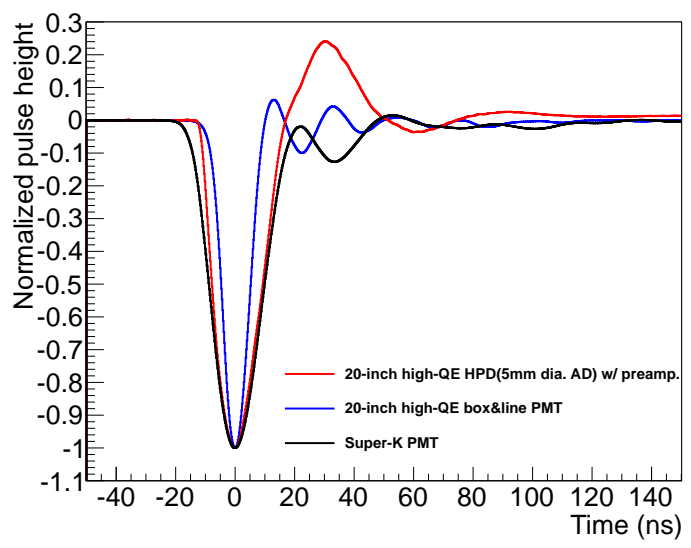

Figure 14: Averaged waveforms of the 20-inch high-QE HPD (red), the 20-inch high-QE box-and-line PMT (blue) and the Super-K PMT (black).

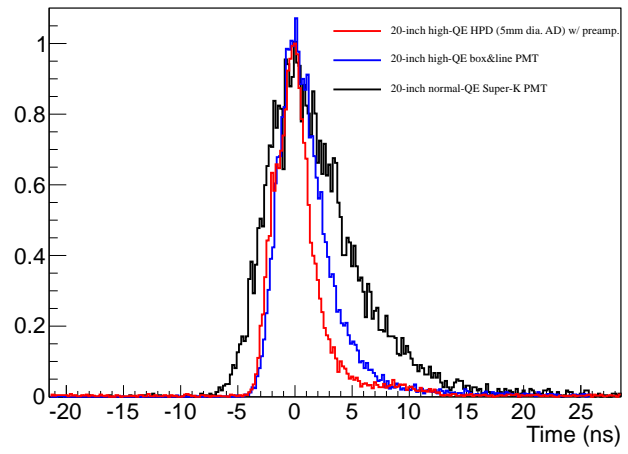

Figure 15: Timing distribution at single photoelectron signal of the 20-inch high-QE HPD (red), the 20-inch high-QE box-and-line PMT (blue) and the Super-K PMT (black).

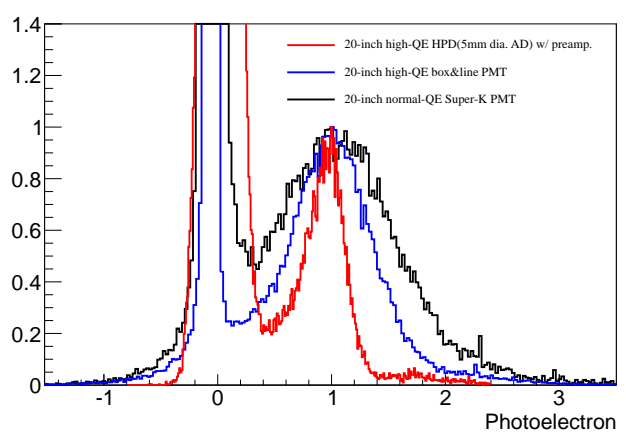

Figure 16: Charge distribution at single photoelectron signal of the 20-inch high-QE HPD (red), the 20-inch high-QE box-and-line PMT (blue) and the Super-K PMT (black).

Table 1: Summary of 20-inch photodetector performance.

\begin{tabular}{|c|c|c|c|}
\hline & $\begin{array}{l}\text { High-QE HPD with } \\
5 \mathrm{~mm} \mathrm{AD}\end{array}$ & $\begin{array}{l}\text { High-QE box-and-line } \\
\text { PMT }\end{array}$ & Super-K PMT \\
\hline Rise time (ns) & 7.4 & 6.2 & 10.6 \\
\hline Fall time (ns) & 11.5 & 6.3 & 13.1 \\
\hline Pulse width (ns) & 25.5 & 16.7 & 31.4 \\
\hline $\begin{array}{l}\text { Timing resolution in } \\
\text { sigma }[\mathrm{FWHM}](\mathrm{ns})\end{array}$ & $1.4[3.4]$ & $1.1[4.1]$ & $2.1[7.3]$ \\
\hline $\begin{array}{l}\text { Single photoelectron } \\
\text { resolution in sigma }(\%)\end{array}$ & 16 & 35 & 53 \\
\hline Peak to valley ratio & 3.9 & 4.3 & 2.2 \\
\hline
\end{tabular}




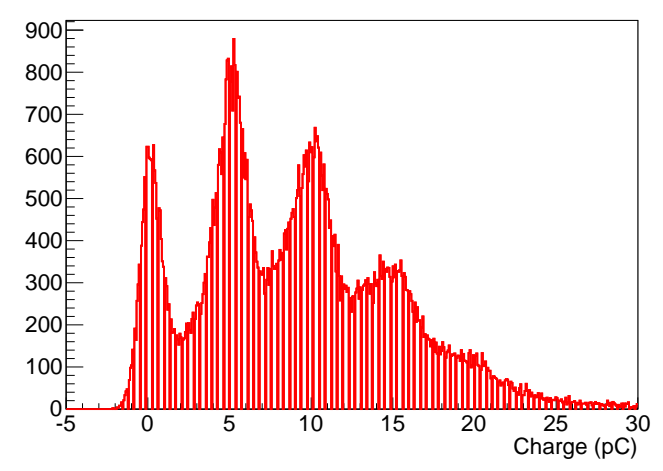

Figure 17: The multi-photoelectron distribution of the 20-inch high-QE HPD.

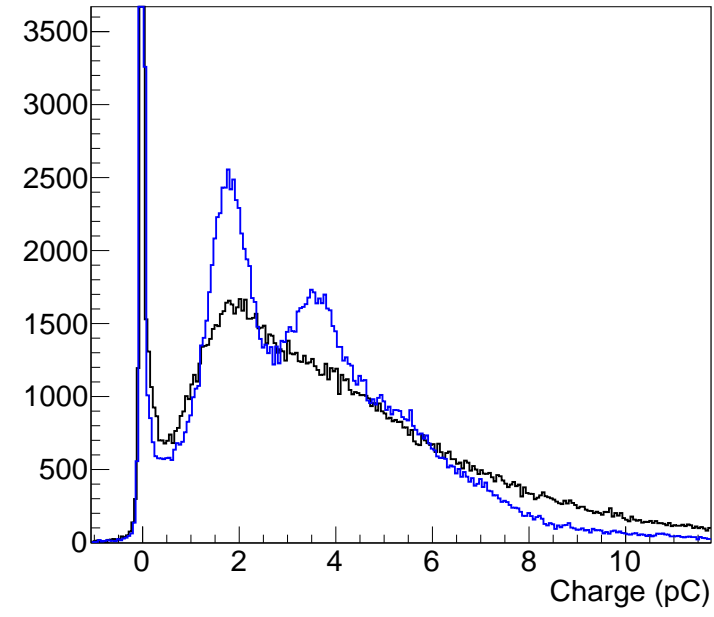

Figure 18: Multi-photoelectron distributions of the 20-inch high-QE box-and-line PMT (blue) and the Super-K PMT (black).

line PMT into the tank and start the test in summer 2014. The 20-inch high-QE HPD will be also tested in the tank in near future. We will complete the R\&D in a few years.

\section{References}

[1] K. Abe et al., "Letter of Intent: The Hyper-Kamiokande Experiment - Detector Design and Physics Potential—, " arXiv:1109.3262 [hep-ex] (2011).

[2] Y. Fukuda et al., "The Super-Kamiokande Detector, " Nuclear Instruments and Methods in Physics Research Section A, 501 418-462 (2003).

[3] Y. Suda, "Performance Evaluation of the Large-Aperture Hybrid Photo-Detector, " Nuclear Instruments and Methods in Physics Research Section A, DOI: 10.1016/j.nima.2014.05.104 (2104).

[4] H. Ikeda et al., "Front End Hybrid Circuit for Super-Kamiokande, " Nuclear Instruments and Methods in Physics Research Section A, 329 299-313 (1992). 